\title{
Examining patterns of injury occurrence in rural and urban settings in sub-Saharan Africa: A Critical Review of the Literature
}

\author{
Daniel Buor, PhD \\ PhD; Professor \\ Department of Geography and Rural Development \\ Kwame Nkrumah University of Science and Technology \\ Kumasi-Ghana
}

\section{Funding}

The research was self-funded.

\section{Author's Contribution}

I am the sole author. No other author contributed to the preparation of this manuscript. Conflict of Interest and Ethical Issue

No conflict of interest and ethical issues were involved.

\begin{abstract}
The paper critically reviews literature on patterns of injury occurrence in rural and urban areas in sub-Saharan Africa. Findings are based on research reports from eight countries purposively selected from major geographical regions in sub-Saharan Africa. From West Africa are selected Nigeria and Ghana; Uganda, Kenya and Tanzania represent East Africa; Rwanda for Central-Eastern Africa; Republic of South Africa for South Africa and Zambia for Central-South Africa. The selected countries are among those where adequate research work on injuries in urban and rural settings has been done. The Sahel region is not listed because it was found that no serious work on injury occurrence by rural and urban has been done in the region. Eight variables, namely, general causes of injury, rate of injury occurrence, severity of injury, persons involved in road traffic injuries (passengers and pedestrians), causes common to both settings, vehicles mostly involved in the injuries, child injuries and age group mostly affected form the basis for the analyses. The review establishes, among other patterns, that rural settings suffer greater severity and higher mortality from injuries through road traffic accidents and occupational (agricultural-related) injuries whilst other injuries including those suffered through pedestrian crossings, burns, cuts and falls are predominant in urban areas. Although a few works tilted the severity of the injuries through motor traffic accidents to the urban areas, the high incidence of occurrence in both rural and urban areas was still upheld. It is recommended, among other considerations, that rural areas be given priority in the establishment of injury clinics whilst the spatial techniques of remote sensing and geographical information systems (GIS), the mixed methods approach and use of inferential statistics be more applicable to capturing and analysing data on injuries.
\end{abstract}

Key Words: injury, sub-Saharan Africa, motor traffic injury, rural, urban, remote-sensing and GIS, mortality.

\section{INTRODUCTION}

Injuries constitute a significant proportion, $16 \%$, of the global burden of disease (World Health Organisation [WHO], 2002). Globally, the injury burden is pronounced especially in developing countries. Injuries are a leading cause of death. The leading causes of death in 2013 for young people aged 10-14 years were HIV/AIDS, road injuries, and drowning (25.2\%) (Mokdad, 
Forouzanfar, Daoud, et al.) In the lower income countries such as those of South Asia and Africa, injury is one of the leading causes of adult mortality and a major contributor to disability in most age groups (Kobusingye et al. 2001; Hofman, 2005; Mock et al. 2014). This translates into 5.8 million injury-related deaths at a rate of 97.7 per 100,000 worldwide. Injuries further account for between 10 and 30 per cent of all hospital admissions and render at least 78 million people disabled each year. The burden of injury in developing countries including African countries is very much pronounced. For instance, 87.9 per cent of all road traffic deaths, and 89.3 per cent of lost disability-adjusted life years (DALYs) are from Lowand-Middle-Income countries [LMICs] (Mathers et al. 2001). Despite the heavy burden from the human immunodeficiency virus and the acquired immune deficiency syndrome (HIV/AIDS), malaria, and other infectious diseases, injuries are still responsible for 19.93 per cent of all deaths of those between the ages of 15 and 59 years (WHO 2002). According to the WHO (2002), road traffic injuries, war and homicide, respectively, were the $10^{\text {th }}, 11^{\text {th }}$, and $14^{\text {th }}$ leading causes of mortality in Africa during 2000. The injury burden is predominant in countries that have emerged from Third World conditions and rural areas are worse affected. Studies by Qing et al. (2012) in China indicate that crude and age-adjusted death rates for 2006-2008 were significantly higher in rural areas than in urban areas. In the United States, injury fatalities are higher in rural counties than urban (Coben, et al. 2004). The injury burden is thus a serious global pandemic. Reasons for the disparity are low development status, rudimentary farming practices, outmoded industrial machines, poor-surface roads and other transport networks, risky mining operations, among others. Low-income countries have the conditions that predispose them to several sources of injury.

Even though several works have been done on the burden of injury in various countries, an attempt to design a model to explain the distribution pattern of injuries by rural-urban in subSaharan Africa has not received much attention. The sub-Saharan Africa region is chosen for critical analysis of the literature due to its economic and social vulnerability. Such an analysis would expose the main causes of the pandemic and unearth mechanisms to address them. It will also bring to the fore inherent gaps and weaknesses in the methodologies used with the intent of recommending more appropriate ones which will result in more realistic results in further works on injury. Using low-income countries as proxy for sub-Saharan Africa, the region could be said to be economically deprived and vulnerable. The economic and social indicators of the region as depicted by the World Bank give evidence of its deprivation and vulnerability. The low gross national income (GNI) [Atlas Method] per capita which is a standard measure of economic wellbeing of a country, average to poor health status indicators, poor physical infrastructure and low participation in education are evidences of the region's deprived status. Life expectancy at birth which is a measure of health status for Uganda, Zambia, Zimbabwe, Mali and Ethiopia which span the sub-regional groupings are 59, 58, 60, 55 and 64 respectively whilst GNI per capita for these countries are US\$600.00, $\$ 1,810.00$, Gh\$860.00, $\$ 670.00$ and $\$ 470.0$ respectively (World Bank, 2015). The low income of most of the countries of the sub-region is largely responsible for the countries' inability to procure adequate relevant security logistics which could help to reduce the injury burden.

The low level of technology of these countries brings in its wake activities such as rudimentary farming practices, especially in the rural sectors, which are associated with injuries. The International Labour Organisation (ILO 2002) reports of workplace accidents mostly in the agricultural sector in developing countries including sub-Saharan Africa. Thousands of workers are killed each year and millions more are either seriously injured in workplace accidents or poisoned with pesticides and other agrochemicals. A large proportion of the injuries in sub-Saharan Africa are caused by road traffic accidents, falls, burns, assaults, bites, 
stings and other related injuries, poisonings, drownings/near-drownings and suicide (Nordberg, 2000).

In rural Africa, injuries and disabilities occur from working with unprotected machinery and lack of or ineffective personal protective equipment at the workplace especially from farms (Amweelo, 2001; Merletti et al., 1998). In urban areas in sub-Saharan Africa the main cause of injuries is road traffic accident (Kobusingye, et al. 2001). Causes such as domestic accidents leading especially to burns, industrial accidents, accidents due to sporting events, violence of all kind, etc. are prevalent and constitute potential sources of injury. Death rates due to injury are generally higher in rural areas than urban areas. Conditions in rural and urban areas in sub-Saharan Africa dictate the types of injury that occur in each of these spatial contexts.

The alarming rate of mortality due to injuries in deprived sub-Saharan Africa calls for a critical review of works done on injuries in rural and urban areas. The study reviews the rural-urban contexts of occurrence of injuries in sub-Saharan Africa using empirical findings of works on injury undertaken by researchers. It answers the question of where, when, how and what of injury occurrence in rural and urban areas in sub-Saharan Africa. It further attempts, in the review, to describe the occurrence of injury in rural and urban areas in selected countries in sub-Saharan Africa with the view to establishing a pattern.

\section{THE CONTEXT}

Sub-Saharan Africa is, geographically, the area of the continent of Africa that lies south of the Sahara desert. Agriculture constitutes over $60 \%$ of the labour force and represents $20 \%$ to $30 \%$ of the GDP and $50 \%$ of exports (The World Bank, 2015). Most agricultural activities are subsistence in which and rudimentary tools are used. Subsistence agriculture, which takes place more in rural settings, exposes farmers to several injury-oriented risks. Oil and mineral extraction, which takes place in several countries of the sub-Region, is a key source of injuries and deaths. Political and ethnic violence has been the lot of sub-Saharan Africa. This which could result in the hypothesis that political and ethnic violence is a function of underdevelopment. Within the past two decades, there has been violence in several spots in sub-Saharan Africa. The Rwandan genocide which claimed over 800,000 lives (Prunier, 1996); the Liberian civil war which claimed over 200,000 lives (Nmoma, 1997); the Sierra Leonean crisis which claimed over 50,000 lives (Anup, 2001), among others, were sources of fatal injuries and injury-related mortalities. There have been other crises such as the Boko Haram insurgency in Northern Nigeria and the Ethiopian crisis, among others, which have cumulatively claimed thousands of lives. All such crises leave in their trail numerous injuries the distribution of which depends on the place of residence, whether rural or urban.

The sub-Saharan Africa environment suffers from some serious environmental problems including deforestation, soil erosion, desertification, wetland degradation, and insect infestation (Mabogunje, 1998). Environmental degradation has affected mostly the rural environment where problems are faced with infrastructural development partly due to uneven terrain. The urban environment is experiencing influx of population from the rural areas (rural-urban migration). The population congestion is thus a recipe for pedestrian accidents and falls.

\section{CONCEPT OF INJURY}

Injury occurs when there is physical damage to the human body as a result of sudden or brief exposure to intolerable levels of energy (Krug 1999; Robertson, 1998; Langley, 2002). It is a term comprising such conditions as fractures, wounds, burns, strains, dislocations, concussions and compressions. It is a damage inflicted on the body by an external force. To the WHO 
injuries are caused by acute exposure to physical agents such as mechanical energy, heat, electricity, chemicals, and ionizing radiation interacting with the body in amounts or at rates that exceed the threshold of human tolerance (WHO, 1992). The types of injuries include abrasion, brain injuries, bruising, burns, cluster headaches, concussions, pinched nerve, pneumothorax and rib fracture (WHO 1992). Injuries can be caused by accidents or acts of violence, and may occur at home, workplace, through playing or when travelling.

Levels of injury are termed severity, which is measured on injury severity scales which are basically used for anatomical descriptions of the injury in respect of the degree of damage to tissues and other body parts. Various injury severity scales such as the International Classification of Diseases (ICD) and Abbreviated Injury Scale (AIS) are widely used in describing the extent of damage caused by injuries (Gennarelli and Wodzin, 2005; Cripton, et al. 2014). The ICD is a general purpose classification of diagnoses and related matters to all health conditions and includes diagnostic codes for both natures of injury and external causes of injury (WHO, 1992). Loo and Anderson (2016) have attempted work on spatial analysis methods of road traffic collisions. The method could be used in the study of rural-urban differences in road traffic collisions and resultant injuries.

\section{MATERIALS AND METHODS}

The paper critically reviews empirical works on rural and urban distribution patterns of injury in sub-Saharan Africa. Eight variables form the basis for the search for rural-urban occurrence. These are: main cause of injury, rate of injury occurrence, severity of injury, age group most affected, persons involved in road traffic injury (passengers or pedestrians), causes common to both rural and urban settings, vehicles mostly involved in road traffic injuries and child injuries. The main cause of injury is selected to give a general picture of injury occurrence in both spaces whilst the age group is to give insight into the potential economic effects of injury in the sub-Region. Child injuries are factored in due to the vulnerability of children to accidents with resultant injuries which would require a special policy direction. Evidences of the above variables are drawn from at least a country from each of the sub-Regional divisions of subSaharan Africa namely East Africa, Central-Eastern Africa, South Africa and West Africa. From West Africa are selected Nigeria and Ghana; Uganda, Kenya and Tanzania represented East Africa; Rwanda from Central-Eastern Africa; Zambia for Central-South Africa and the Republic of South Africa for South Africa. The selected countries are among those where adequate research work on injuries in urban and rural settings have been done. The Sahel region is not listed because it was found that no serious work on injury occurrence by rural and urban has been done in the region. The WHO has some general data on injury occurrence in the Sahel region but not on rural-urban distribution pattern. Several works from each of the regions are assembled and the most representative of the findings from the group of works is selected for each region. Data used was taken from published research reports for the last two decades.

\section{ANALYSIS OF WORKS FROM SELECTED COUNTRIES}

Results through literature search are analysed for each country. Results for works done in each country are related to see convergences and divergences. The methods used are critiqued and recommendations on more comprehensive methods to ensure adequate data for a more comprehensive analysis made. Recommendations to improve injury control in both settings are also made.

\section{Ghana}

The work on road traffic injuries in Ghana by Afukaar, et al. (2003) presents a crystal picture of the injury situation in Ghana. Authors analysed road traffic injuries, which are the main cause of fatalities, and injuries in most parts of sub-Saharan Africa. Police-collected crash in injury 
data for the period 1994-1998 were aggregated and analysed using the MAAP 5 accident package developed by the Transport Research Laboratory. Road traffic crashes were found to be a leading cause of death and injuries in Ghana. The other leading causes of death and injuries were occupational injuries, which involve non-mechanised farming and tribal conflicts. Majority of road traffic fatalities and injuries occurred on roads in rural areas. About 58\% more people died on roads in rural areas than in urban areas and pedestrians accounted for $46.2 \%$ of all road traffic fatalities with majority of the pedestrians' fatalities occurring in urban areas. Findings are corroborated by the work of Mock et al. (1999) in their paper on epidemiology of transport related injuries in Ghana. They found the incidence of transport reported injuries to be identical in both rural and urban settings. In both settings, transport reported injuries were more severe than other types of injuries in terms of mortality, length of disability, and economic consequences. Whereas in the urban areas the most common transport-related mechanisms were either to passengers involved in crashes of mini buses or taxis or to pedestrians struck by these vehicles in the rural areas, the most common transport related mechanisms were bicycle crashes.

Abantanga and Mock (1998) examined childhood injuries in an urban area of Ghana in a hospital-based study of 677 cases. The most common mechanism of injury, as in the previous surveys by Afukaar et al. (2003) and Mock (1999), were pedestrian knock downs (40\%), falls $(27.2 \%)$, and burns (17.6\%) and boys sustained high injury rates in all age groups than girls. In analysing the region of principal injury, for severe injuries with abbreviated injury scale of 3-5, the extremities suffered most, followed by the skin and head were established. There was an injury severity score of $>20$. The study did not make reference to rural conditions.

In a study of pedestrians' injury patterns in Ghana, Damsere-Derry (2009) in a retrospective analysis of de-identified pedestrian crash data between 2002 and 2006 found that the main causes of pedestrian deaths and injuries were crossing the road (over 78\%), excessive speeding (65\%) and driver attentiveness. Pedestrians were less likely to die when struck by private cars (52\%), pick up trucks (57\%), and motorcycles (86\%) compared to buses. The study did not compare rural-urban disparities but confirms studies by Abatanga and Mock (1998), Afukaar et al. and Mock (1999) on urban incidence and severity and cause of injuries and fatalities. This study was not a comparative one so did not establish differences between rural and urban. The urban features, nevertheless, strengthen works already done.

In a study on incidence and outcome of injury in Ghana: a community-based survey, Mock et al. (1999), using a two-stage cluster sampling and household interviews, the findings confirmed other studies on injury carried out on Ghana. Injury-related mortality was slightly higher in the urban (83 per 100,000) than in the rural area (53 per 100,000). However, the burden disability from nonfatal injuries, as assessed by disability days was higher in rural (4,697 disability days per 1000 person-years) than in the urban area (2671 days per 1000 person-years). Whereas the major types of injury in the urban area were transport-related injury and falls, in the rural area, agricultural injuries predominated, followed by falls and transport-related injury. In rural and urban areas combined, $73 \%$ of motor vehicle-related injuries involved commercial vehicles. This work would have been enhanced by the use of remote-sensing and geographical information systems (GIS); scientific techniques for capturing spatial information. Besides, the use of the mixed methods approach would have established views and opinions about road traffic accidents and resulting injuries (O'Cathaian et al. 2008; Cameron, 2013). Respondents could give a vivid description of how they see injuries in both settings and mechanisms to address them. 


\section{Nigeria}

A South-Western Nigeria study added the sex dimension of injury occurrence to its objectives. A study of incidence and pattern of injuries among residents of a rural area in South-Western Nigeria: a community-based study, Omoniyi and Eme (2007) established that injury incidence for females (46 per 1000 per year) was lower for males which was 159 per 1000 per year. The other studies did not concentrate on demographic and socio-economic characteristics of the injured. Falls and traffic injuries were the leading causes of injury, followed by cuts and blunt injuries. Traffic injuries were the leading cause of injuries in all age groups except among the 514 years where falls were the leading cause of injury. Thus after traffic-related injuries, falls come next in injury occurrence. Traffic or transport related injuries have been found to be the main cause of injuries in Nigeria. Ekere (2005) confirms this is a study of mortality patterns in the accident and emergency department of an urban hospital in Nigeria.

A population-based survey on the burden of traffic injuries carried out by Labinjo, et al. (2009) established that road traffic injury rates for rural and urban areas were not significantly different. The rates for both rural and urban areas are high motorcycle crashes accounted for $54 \%$ of all road traffic injuries. The situation may be due to the large population and increasing rate of urbanisation. Increased risk of injury was associated with male gender among those aged 18-44 years, with a relative risk of 2.96 when compared with women in the same age group whilst. This result confirms other in the selected countries. This work would have enjoyed more credible results with the use of the mixed methods/triangulation approach (O'Cathaian et al. 2008; Cameron, 2013). This approach would have satisfied the pragmatic philosophy. Besides, the use of remote-sensing and GIS as research tools are more credible mechanisms for deriving information on occurrence of accidents and resulting injuries.

\section{Tanzania}

Studies in Tanzania confirm some patterns in the occurrence and severity of injuries in West Africa. A study of injury morbidity in an urban and a rural area in Tanzania: an epidemiological survey, Moshiro et al. (2005) made some observations. Firstly, the overall incidence of injuries was higher in the rural area whilst the incidence of major injuries was similar in both areas. Secondly, males were at a higher risk of having an injury than females. Thirdly, rural residents were more likely to experience injuries due to falls and cuts but had a lower risk of transport injuries. The most common causes of injury in the urban area were transport injuries and falls. In the rural area, cuts and stabs, of which two thirds were related to agriculture, formed the most common cause of injuries. This work should have been grounded on a theoretical foundation and a more scientific approach and the use of remote sensing and GIS to capture spatial information.

\section{Kenya}

Odero, et al. (2010) writing on road traffic injuries in Kenya: magnitude, causes and status of intervention established that more than $75 \%$ of road traffic casualties are economically productive young adults and pedestrians and passengers are the most vulnerable. Pedestrians are more likely to be killed in urban areas, whereas passengers are the majority killed on intercity highways in rural settings. Injuries follow the same pattern with more injuries occurring through motor accidents that affect pedestrians than in rural settings. On the other hand, there are more injuries incurring through motor accidents affecting passengers. Thus, it runs through all the studies that whereas road accident injuries that affect passengers have a high incident rate in the rural settings, there are more injuries through motor knockdowns of pedestrians in urban areas. In this study, there was reliance on police data as in some other studies. It is relevant to state that the use of the mixed methods-triangulation approach must be emphasised to get the opinions and eyewitness account of such occurrences in the 
communities. The use of the GIS and remote-sensing techniques must also be emphasised in order to ensure storage of spatial data on accidents and resulting injuries for planning purposes and making policies to reverse the incidence of accidents and injuries.

\section{Uganda}

The Ugandan studies showed results different form the other countries with regard to the concentration of injuries sustained by adults. In a study on injury patterns in rural and urban Uganda, Kobusingye et al. (2001) using the interview approach in a household survey observed that the urban population is at a higher risk than rural population in the injury burden. Whereas Mukomo rural district recorded an annual injury prevalence rate of $0.7 \%$, that of Kawempe, a geopolitical division of Kampala was $2.8 \%$. In all age groups 20 years and older in Mokono rural district, traffic was the most important cause. Traffic was the major cause of injuries in the Kawempe urban district as well. Falls were the most common cause of severe injuries in children 10 years and younger in the rural district whilst for the urban district it was burns. The concentration in the urban areas may be the outcome of population distribution by rural-urban. The study, notwithstanding, establishes the primacy of road traffic accidents in both rural and urban areas as observed by studies in other regions.

\section{Rwanda}

Not much research on injuries has been engaged in Central-Eastern Africa as in other regions in sub-Saharan Africa and in this region research has concentrated on specific socio-economic and demographic variables. In a study of the epidemiology of road traffic injury in Kigali, Rwanda using police data, Patel et al. (2016) observed that the majority of injury victims were male with an average age of 35.9 years and cars were the most frequent vehicle involved followed by motorcycles with pedestrians and cyclists being more likely to have grievous injuries. Most of these findings correspond with other findings in other empirical studies in the sampled countries in sub-Saharan Africa. The use police injury data is phenomenal. It is indeed the standard practice in assessing works on accidents and injuries. Inputs of respondents in the communities on the frequency of accidents and associated injuries have been side-lined. It is pertinent to note that, since accidents and their resulting fatalities are social phenomena, the perspectives of causes and implications will call for inputs from the communities. It is not uncommon for people to rush to accident scenes. Sometimes even before the police teams arrive, people from the communities would have had first hand information about the occurrences.

\section{South Africa}

Studies in South Africa have concentrated on injury deaths and child mortality due to injuryrelated accidents. A work on a comparison of urban-rural injury mortality rates across two South African provinces, Sherriff (2007) found that, homicide and unintentional (nontransport) injury death rates were significantly higher in the urban province than the rural whilst transport-related injury mortality rates were significantly higher in the rural province than the urban. There are more fatal road crashes in rural than urban areas and more injuries recorded in rural than urban areas (Bartley, 2008). Besides, there are more pedestrian deaths through road traffic injury than passengers. Moreover, homicide ranks high in injury deaths, with males outnumbering females in the episodes. In a study of urban and rural differences in child injury deaths in South Africa: A one year review, Swart et al. (2012) established that overall injury death rates among children were higher among urban Gauteng children than rural Mpumalanga children and passenger-related motor vehicle deaths were more evident among children in rural areas than in urban areas. Unintentional (non-transport-related) deaths-specifically those associated with burns-were more common among urban children 
than among rural children. Such differences may arise because of the many environmental and infrastructural-related differences that exist between rural and urban areas.

\section{Zambia}

Seidenberg, et al. (2014) examining epidemiology of injuries, outcomes, and hospital resource utilisation at a tertiary teaching hospital in Lusaka, Zambia, observed that injury patients are primarily male and majority of injury incidents were through private vehicle and public transport. The most common reported mechanisms of injury were falls, road traffic accidents and assault. This research did not capture the broad dimensions of the socio-demographic and economic characteristics of the respondents for purposes of planning. The use of inferential statistics would have provided a stronger basis for making more realistic conclusions and generalisations than the resort to descriptive statistics.

\section{DISCUSSION AND CONCLUSIONS}

The paper has attempted a critical review of empirical works on patterns of injury occurrence in rural and urban areas in sub-Saharan Africa. It is evident in the analysis that rural areas suffer more from the trauma of injuries than urban areas and the burden of motor traffic injuries is predominant in both the rural and urban areas. It is also evident that pedestrians suffer greater injuries in the urban areas whilst passengers suffer the same in rural areas. Injury occurrence has a demographic pattern. Whereas males suffer greater injuries and deaths through traffic injuries and homicide in both rural-urban settings, children in rural areas suffer more from road traffic injuries whilst those in urban areas suffer more from nontraffic (unintentional) injuries such as falls, burns and cuts. The rural environment, which has poor facilities and less security from road traffic accidents, is more prone to overall injuries than their urban counterparts. This is the pattern in some developed countries including Ireland (Boland, et al. 2005) and United States (Jeffrey, 2004). It is reflected by some works in some other developing countries outside sub-Saharan Africa. Ghaffar (2004) working on the burden of traffic injuries in developing countries: the $1^{\text {st }}$ national injury survey of Pakistan noted this. Road infrastructure and other infrastructure such as electrical installations and even housing facilities in the rural areas are more fragile and prone to environmental hazards than those in the urban areas. Besides, they suffer predominantly from agricultural injuries; from using rudimentary tools, chemicals without safety gadgets, snakebites due to none or inadequate materials for body protection whilst working on the farm, and falls. This smacks of developmental implications. Rural areas which lack facilities to address injury problems rather suffer more from injuries. This is an irony, which lies at the basis of the spatial developmental dichotomy, which must be addressed. More resources must be channelled to the rural areas in the fight against the injury burden. In the urban areas children suffer more from non-traffic related injuries such as falls, burns and cuts. Kim (2012) made similar observations in his work in Canada. Children are a vulnerable group so need attention to safe them from preventable injuries.

Works on occurrence of rural and urban patterns of injury in sub-Saharan Africa generally has some drawbacks. Firstly, some works do not use the remote-sensing and GIS methodology in capturing information on injury occurrence. The use of this scientific method will place the occurrence of the events in a proper perspective. Secondly, for a balanced research, the mixed method approach gives a proper picture of reality but this does not reflect in most of the works. Life experiences give a better picture of events than the quantitative approach. Moreover occurrences more often than not have not taken into consideration the demosocioeconomic characteristics of victims. Besides, it is recommended that the multivariate statistical approach, making inferences would better address the objectives of such research. The use of the descriptive statistical techniques has been predominant in works on accidents 
and injuries. The socio-economic implications of such events could best be measured using a more scientific approach with the use of inferential statistics.

It is recommended that in urban areas hospitals and clinics to address injuries be established rather than burns and injuries departments to be joined to the main hospitals. With road traffic injuries being predominant, more resources must be channelled to this area. Special casualty units must be created in rural hospitals. Road safety measures must be implemented. Road safety measures often include information and education campaigns, improved driver training, road design and maintenance, regular vehicle safety checks, separation of pedestrians from vehicle traffic, speed limits, safety belt, air-bag and helmet use, special training and control of public service vehicle drivers, bicycle lane separation, road lighting, reflectorized materials on clothing, review of the road traffic related legislation and law enforcement, and emergency vehicular services improvement (Nordberg, 2000). Sanctions for over speeding and alcoholism of drivers must be applied rigidly whilst the problem of fading or non-existent road traffic signs be addressed. Besides, some police officials who allegedly err in enforcing road traffic regulations due to corruption must be sanctioned. Moreover, the use of remote sensing and GIS in capturing information gives a truer picture of the spatial perspectives (Maryam, 2017) of occurrence of injury in the two settings. Finally, a combination of the qualitative and quantitative designs in analysing data on injuries will give a better representation of the pragmatic philosophy.

\section{References}

Abantanga FA and Mock, CN (1998) Childhood injuries in an urban area of Ghana: A hospital-based study of 677 cases. Pediatric Surgery International, 13(7), 515-518.

Afukaar FK, Antwi P, and Ofosu-Amaah S (2003) Pattern of road traffic injuries: Implications for control. Injury Control and Safety Promotion, 10(1-2), 69-76.

Amweelo M (2000) Occupational health and safety problems in Namibian agriculture. African Newsletter on Occupational Health and Safety, 11 (2), 44-47.

Anup S (2001) Sierra Leone. Global Issues. SAGE Publishers: New York

Bartley GP (2008) Causes of road traffic accidents in developing countries. Bartley GP (Ed), Traffic Accidents: Causes and Outcomes. Nova Publishers: New York.

Boland M, Staines A, Fitzpatrick P, Scallan E, (2005) Urban-rural variation in mortality and hospital admission rates for unintentional injury in Ireland. Journal of Epidemiology and Community Health, 59: 557.

Cameron, R. (2013), Mixed Methods Research: a world of metaphors', in Metaphors for, in and of Education Research, (Eds) Midgley, W; Trimmer, K. and Davies, A. (2012). Chapter 4, pp. 51-65. Cambridge Scholars, Newcastle upon Tyne, UK. ISBN: 1-4438-43908-9.

Coben JH, Tiesman HM, Bossarte RM, Furbee PM, (2004) Rural-Urban differences in injury hospitalisation in the US. American Journal of Preventive Medicine, 36(1), 49-55.

Cripton PA, Dressler DM, Stuart CA, Dennison CR, Richards D, (2014) Bicycle helmets are highly effective at preventing head injury during head impact: Head-form accelerations and injury criteria for helemeted and unhelmeted impacts, Accident Analysis and Prevention, 70, 1-7.

Damsere-Derry, Beth EE, Donkor P (2010) Pedestrians injury patterns in Ghana. Accident Analysis and Prevention 42(4),1080-1088.

Ekere AU, Yellowe BE, Umunne S (2005) Mortality patterns in the accident and emergency department of an urban hospital in Nigeria. Nigerian Journal of Clinical Practice, 8(1), 14-18.

Genarelli TA, Wodzin E (2005) Abbreviated Injury Scale 2005.

Ghaffar A, Hydei AA, Masud TI, 2004 The burden of traffic injuries in developing countries: the $1^{\text {st }}$ national injury survey of Pakistan, Public Health 118(3), 211-217.

Hofman K, Primack A, Keusch G, Hrynkow S, (2005) Addressing the growing burden of trauma and injury in lowand middle-income countries. American Journal of Public Health 95, 13-17. 
International Labour Organisation (ILO) (2002) Safe Work: Occupational Safety and Health in Agriculture. http://www.ilo.org.

Jeffrey HC, Hope MT, Bossarte RM, Paul, MF (2004) Rural-Urban differences in injury hospitalisations in the U.S. American Journal of Preventive Medicine DOI:http//dx.dfoi.org/10.1016/jk.amepre.2008.10.001.

Kim K, Ozegovic D, Voaklander DC (2012) Differences in incidence of injury between rural and urban children in Canada and the USA: a systematic review. Injury Prevention 18(4), 264-271.

Kobusingye O, Guwatudde D, Lett R (2001) Injury patterns in rural and urban Uganda, Injury Prevention 7(1), 4650 .

Kobusingye 0, Guwatudde D, Owor G, Lett RR (2002) Citywide trauma experience in Kampala, Uganda: a call for intervention, Injury Prevention 8, 133-136.

Labinjo M, Juillard C, Kobusingye OC, Hyder AA (2009) The burden of road traffic injuries in Nigeria: results of a population-based survey Injury Prevention 15, 157-162.

Loo PYB, Anderson TK (2016) Spatial Analysis Methods of road traffic collisions, Boca Raton, USA.

Mabongunje AL (1998) The environmental challenges in sub-Saharan Africa African Technology Forum, Massachusetts Institute of Technology, 8 (1).

Maryam, A., Ali, V., Ali, M. 2017. Geographic information system (GIS) capabilitiues in traffic accident information management: a qualitative approach, Electron Physician. 9(6): 4533-4540.

Matthers CD, Sadana R, Salamon JA, Murray CJL, Lopez AD (2001) Healthy Life Expectancy in 191 Countries, 1999 Lancet 1685-1691.

Merletti F, Soskolne CI, Vineis P (1998) Epidemiological methods applied to occupational health and safety, Encyclopaedia of Occupational Health and Safety, $4^{\text {th }}$ Edition, ILO, Geneva.

Mock CN (1999) Epidemiology of transport-related injuries in Ghana Accident Analysis and Prevention 31(4), 359370.

Mock CN, Abantanga F, Cummings P, Koepsell TD, (1999) Incidence and outcome of injury in Ghana: a communitybased survey Bulletin of the World Health Organisation 77(12), 955-964.

Mock CN (2001) Trauma system development in Ghana Ghana Medical Journal 35,1- 3.

Mock C, Quansah R, Kobusingye 0, Goosen J (2014) Trauma care in Africa African Journal of Trauma 3(1), 3-10.

Mokdad AH, Faropuzanfar MH, Daoud F et al. (2016) Global burden of diseases, injuries, and risk factors for young people's health during 1990-2013: a systematic analysis for the Global Burden of Disease Study 2013. Lancet 11, 387(10036), 2383-401.

Moshiro C, Ivar H, Nordrehaug A.A., Setel P, Hemed Y, Kvale G (2005) Injury morbidity in an urban and a rural area in Tanzania: an epidemiological survey BMC Public Health, 10.1186/14571-2458-5-11.

Nmoma V, 1997 The civil war and the refugee crisis in Liberia The Journal of Conflict Studies xvii (1).

Nordberg E (2000) Injuries as a public health problem in sub-Saharan Africa: epidemiology and prospects for control East African Medical Journal 77 (12 Supplement), S1-43.

O'Cathaian, A., Murphy, E., Nicholl, J. 2008. The quality of mixed methods studies in health services research. Journal of Health Services Research Policy, 13:92-98.

Omoniyi AO, Eme, T.0. 2007. Incidence and pattern of injuries among residents of a rural area in South-Western Nigeria. BMC Public Health, 10-.1186/1471-24568-7-246.

Patel, A., Krebs E, Luciano A, Rulisa S, Joao RNV, Stato CA (2016) Epidemiology of road traffic injury hotspots in Kigali, Rwanda from police data Public Health BMC 16,697. DOI:10.1186/s128899-016-3359-4.

Prunier G (1996) The Rwandan Crisis: History of a Genocide. Columbia University Press.

Qing L, Lan Z, Qingjun Z (2012) The gap in injury mortality rates between urban and rural residents of Hubei province, China. BMC Public Health 12,180.

Robertson LS (1998) Injury Epidemiology, $2^{\text {nd }}$ Edition, New York: Oxford University Press, 265.

Seidenberg P, Oranmore RB, Hammond E (2014) Epidemiology of injuries, outcomes, and hospital resource utilisation at a tertiary teaching hospital in Lusaka, Zambia. African Journal of Emergency Medicine, 4(3),115-122. 
Buor, D. (2018). Examining patterns of injury occurrence in rural and urban settings in sub-Saharan Africa: A Critical Review of the Literature. Advances in Social Sciences Research Journal, 5(12) 182-192.

Sheriff B, MacKenzie S, Swart LA, Seedat MA, Bangdiwala SI, Ngude R (2007) A comparison of urban-rural injury mortality rates across two South African provinces International Journal of Injury Control and Safety Promotion, $222(1), 75-85$.

Swart LA, Laher H, Seedat M, Gantchev G (2012) Urban and rural differences in child injury deaths in South Africa: a one-year review African Safety Promotion Journal 10 (2): 28-40.

Taub FB (1993) Book Review: Estimating ecological risks Ecology 74,1290-1291.

White GC, Anderson DR, Burtnham KP, Otis DL, (1982) Capture-recapture and removal methods for sampling closed populations Los Alamos National Laboratory, LS-8787-NERP, Los Alamos, NM, USA.

World Bank (2015) World Development Indicators, Washington DC.

WHO (1992) ICD-10 International statistical classification of diseases and related health problems Volume 1. WHO: Geneva. 1-1243.

WHO (2002) World Report on Violence and Health Geneva: Switzerland.

WHO (2010) World Health Statistics Geneva: World Health Organisation. 